Ann. Biol. anim. Bioch. Biophys., 1978, 18 (1), 119-126.

\title{
Effets de l'ablation du corps ultimobranchial et de la perfusion de calcitonine sur les flux de calcium au niveau des branchies de l'anguille (Anguilla anguilla L.)
}

\author{
par Jacqueline PEIGNOUX-DEVILLE, C. MILET, Elizabeth MARTELLY \\ Laboratoire de Physiologie générale et comparée, Muséum national d'Histoire naturelle, \\ Laboratoire d'Endocrinologie comparée associé au C.N.R.S., 7, rue Cuvier, 75005 Paris.
}

Summary. Effects of ablation of the ultimobranchial body and of calcitonin perfusion on calcium influx and outflux in the gills of the eel Anguilla anguilla $L$.

The effects of calcitonin (CT) perfusion and removal of the ultimobranchial body (UB) upon calcium transfer in the gills of the eel Anguilla anguilla L. were studied with an unidirectional calcium flux measurement method on an isolated gill arch perfusion preparation. $C T$ reduced calcium influx and increased calcium outflux. The reduction of $\mathrm{Ca}$ influx after removal of the UB was due to the effect of activated Stannius corpuscles. We have shown that gill response to $C T$ was quantitatively modified by ultimobranchialectomy. These influx and outflux changes were consistent with an hypocalcemic effect of $C T$ in fish and demonstrated that the gill is a target organ for this hormone.

\section{Introduction.}

Le système endocrinien impliqué dans la régulation du métabolisme calcique chez les Poissons est très différent de celui des Tétrapodes. Les parathyroïdes n'ont jamais pu être mises en évidence chez les Poissons (Fleschman, 1947 ; Hoar, 1951 ; Pickford, 1953) ; certains d'entre eux (Holostéens et Téléostéens) possèdent des glandes originales décrites par Stannius en 1839, les corpuscules de Stannius (CS) qui interviennent dans la régulation du métabolisme calcique (Fontaine, 1964, 1967 ; Chester-Jones et al.., 1965 ; Fontaine et al., 1972). Le corps ultimobranchial (CUB) sécrétant la calcitonine (CT) apparaît chez les Chondrichthyens et est présent ensuite dans toute la lignée des Vertébrés. On sait aussi que le foie de certains Téléostéens contient de grandes quantités de vitamine $D$ (Bills ef al., 1954).

L'ablation chirurgicale du CUB chez l'Anguille provoque une hypercalcémie post-opératoire (Lopez ef al., 1976). Celle-ci est toutefois moins importante que celle observée après ablation des corpuscules de Stannius (Fontaine, 1964). Dans les deux cas on note un maximum se situant environ deux semaines après l'opération puis un refour à la normale du taux de calcium sanguin (Fontaine, 1964 ; Milet et Martelly, résultats inédits). L'éłude des flux de calcium branchiaux, chez les animaux privés de CS, fait apparaître des modifications de ces flux en relation avec la teneur en calcium 
du sang, c'est-à-dire aussi en relation avec le temps écoulé depuis l'opération. Le sens de ces modifications (diminution du flux d'entrée et augmentation du flux de sortie) explique en partie le retour à la normale de la calcémie après ablation des CS (Milet et al., 1975). Le corps ultimobranchial présentant des signes histologiques de stimulation sécrétoire après ablation des CS (Lopez ef al., 1968), on peut supposer que l'hypercalcémie consécutive à cette opération chirurgicale serait le facteur de stimulation de cette glande et que la CT ainsi sécrétée agirait au niveau branchial, tendant à restaurer la calcémie.

Pour nous permettre de vérifier cette hypothèse il était nécessaire de mettre en évidence le rôle de la CT sur les flux de calcium branchiaux par ablation du CUB et par perfusion de CT. Nous avons, dans ce travail, recherché les effets sur les flux branchiaux de calcium, d'une part de l'ablation du CUB, d'autre part de la CT perfusée in vitro.

\section{Matériel et méthodes.}

L'étude du transport branchial de calcium est faite sur des Anguilles argentées femelles immatures d'un poids moyen de $450 \mathrm{~g}$. Les animaux sont divisés en deux groupes:

- ceux du premier groupe subissent l'ablation du CUB selon une méthode dérivée de celle de Chan (1972) et améliorée au laboratoire de façon à ce que l'opération se déroule sans aucune hémorragie. L'animal estanesthésié au MS 222 ( 3 g pour 7 I d'eau). Une incision de 1,5 à $2 \mathrm{~cm}$ est pratiquée ventralement entre les deux nageoires pectorales. Le cœur est repoussé sur le côté et le tube digestif est incisé sur quelques millimètres. Un thermocautère courbe est introduit dans le tube digestif pour cautériser la région constiłuée par du tissu conjonctif, contenant le CUB. Le schéma (fig. 1) montre que nous ne provoquons aucun dommage aux organes vitaux voisins du CUB. - les animaux du second groupe sont opérés témoins c'est-à-dire qu'ils subissent l'opération décrite ci-dessus, sauf la cautérisation.

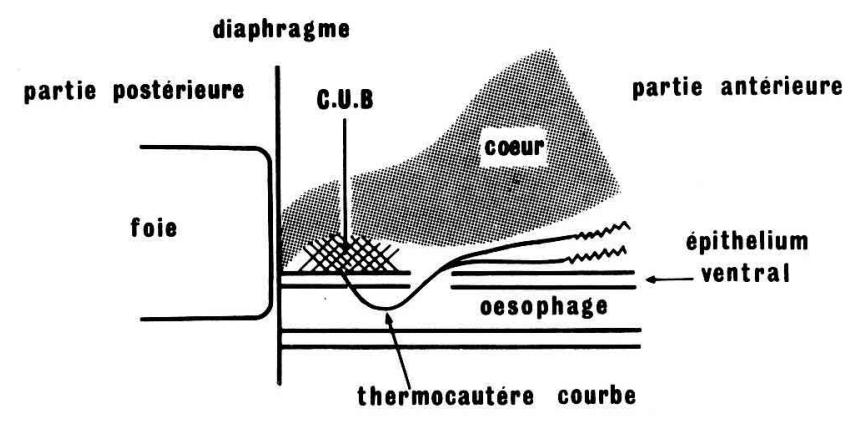

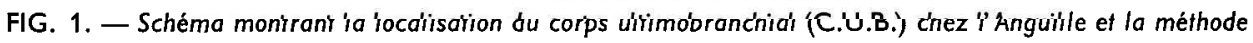
de cautérisation employée pour l'ultimobranchialectomie.

Les mesures de flux de calcium sont réalisées in vitro, une semaine après l'opération, sur une préparation d'arc branchial isolé et perfusé, technique mise au point au laboratoire par Milet et Martelly (en préparation). Cette méthode consiste à perfuser 
une branchie isolée avec du Ringer composé de : $\mathrm{Na} \mathrm{Cl} 136 \mathrm{mM}, \mathrm{Ca}^{++}\left(\mathrm{Ca} \mathrm{Cl}_{2}\right)$ $3 \mathrm{mM}, \mathrm{K} \mathrm{Cl} 2 \mathrm{mM}, \mathrm{Mg} \mathrm{SO}_{4} 1,9 \mathrm{mM}$, glucose $3 \mathrm{mM}$, tamponné à $\mathrm{pH}$ 7,4 par du tampon phosphate $0,2 \mathrm{M}$ et filtré sur millipore $0,25 \mu$ avant son utilisation.

Sur une première préparation, pour la mesure du flux d'entrée (fe), un traceur radioactif ${ }^{45} \mathrm{Ca}$ est introduit dans le compartiment externe dans lequel baigne la branchie et est recueilli à la sortie de la branchie ; la mesure du ${ }^{45} \mathrm{Ca}$ dans le perfusat permet de calculer le fe de calcium au niveau branchial. Sur une préparation, le traceur radioactif ${ }^{45} \mathrm{Ca}$ est ajouté au liquide de perfusion et recueilli dans le compartiment externe où sa mesure permet de calculer le flux sortant ( $\mathrm{fs}$ ) de calcium. L'expérience est réalisée dans de l'eau, bullée par un mélange 95 p. $100 \mathrm{O}_{2}$; 5 p. $100 \mathrm{CO}_{2}$, à température constante $\left(12{ }^{\circ} \mathrm{C}\right)$ et dont le taux de calcium est d'environ $80 \mathrm{mg} / \mathrm{l}$.

Une étude approfondie de la vascularisation branchiale a permis de réaliser la préparation utilisée lors de cette expérience. Le débit de perfusion a été calculé après mesure du débit sanguin d'un arc branchial in situ. Six arcs d'un même animal sont prélevés : trois pour la mesure du flux d'entrée, les trois autres pour celle du flux de sortie. Les mesures ont été faites sur 18 arcs branchiaux d'Anguilles opérées témoins et sur 26 arcs branchiaux d'Anguilles ultimobranchialectomisées. Les flux de base sont déterminés pendant les 35 premières minutes de perfusion. La calcitonine (CT synthétique de Saumon *) à raison de $20 \mathrm{ng} / \mathrm{ml}$ de Ringer, est ensuite perfusée pendant le reste de l'expérience, soit $1 \mathrm{~h}$. Cette concentration a été choisie d'après le taux circulant de cette hormone chez le Saumon (Watts ef al., 1975). En fait, d'après une publication récente, le taux de la CT circulante chez l'Anguille est plus faible $(1,5 \mathrm{ng} / \mathrm{ml})$ (Orimo et al., 1976).

Les flux de calcium exprimés en $\mu \mathrm{Eq} / \mathrm{h} / \mathrm{kg}$ d'animal sont calculés à partir de la pente de la droite expérimentale représentant l'accroissement de la radioactivité mesurée en fonction du temps. Cette quantité de radioactivité est convertie en quantité équivalente de Ca stable en utilisant les formules suivantes :

$$
\begin{aligned}
& \text { flux d'entrée en } \mu \mathrm{Eq} / \mathrm{h} / \mathrm{kg}: \frac{\mathrm{cpm} / \mathrm{min} \text { (dans le perfusat) } \times 60 \times 10^{6}}{\text { RAS du milieu } \times \text { pds du corps } \times 20} \text {. } \\
& \text { flux de sortie en } \mu \mathrm{Eq} / \mathrm{h} / \mathrm{kg}: \frac{\mathrm{cpm} / \mathrm{min} \text { (dans le milieu) } \times 60 \times 10^{6}}{\text { RAS Ringer } \times \text { pds du corps } \times 20} .
\end{aligned}
$$

\section{Résultats.}

Chez les Anguilles opérées témoins, la CT in vitro provoque une diminution faible mais significative du flux d'entrée de calcium à travers la branchie et une augmentation importante du flux de sortie (fig. 2). Les valeurs des flux sont données dans le tableau 1.

* La CT synthétique de Saumon nous a été aimablement fournie par les Laboratoires SANDOZ que nous remercions. 

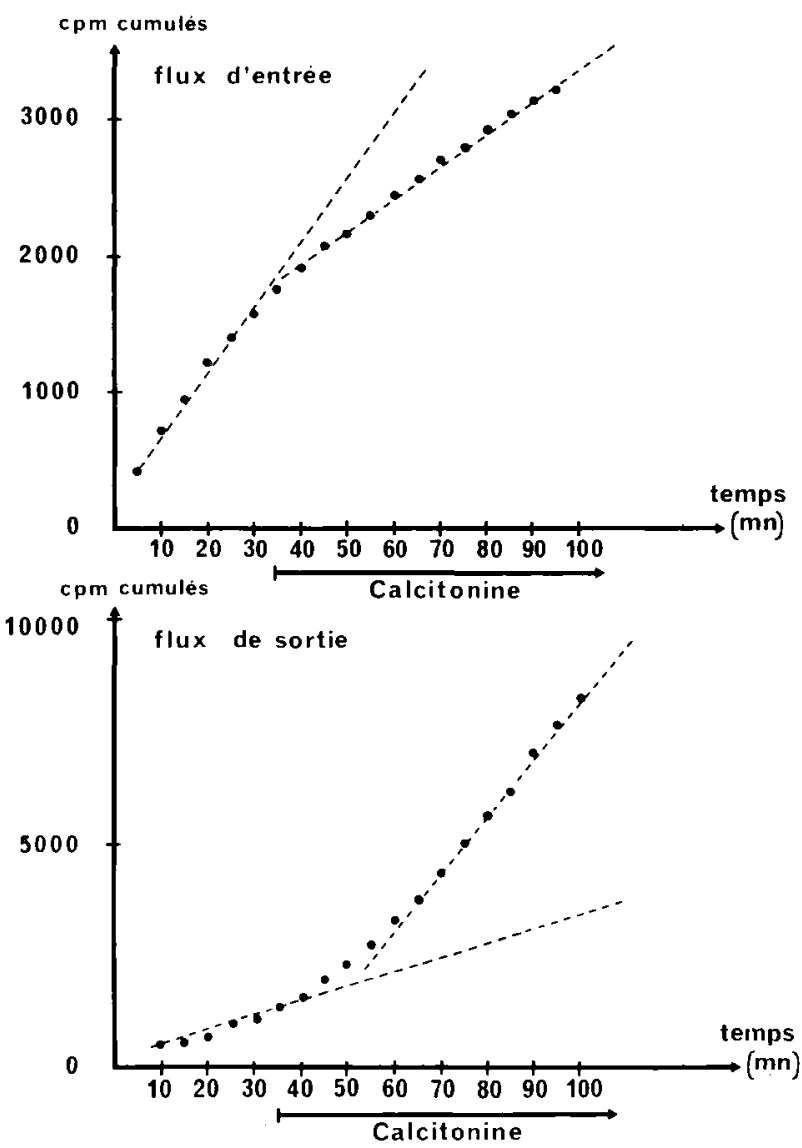

FIG. 2. - Effet de la perfusion de calcitonine sur les flux de calcium dans les arcs branchiaux d'Anguille.

\section{TABLEAU 1}

Action de la CT sur les flux branchiaux de calcium chez l'Anguille femelle argentée

\begin{tabular}{|c|c|c|}
\hline & $\begin{array}{c}\text { Flux d'entrée } \\
\mu \mathrm{Eq} / \mathrm{h} / \mathrm{kg}\end{array}$ & $\begin{array}{c}\text { Flux de sortie } \\
\mu \mathrm{Eq} / \mathrm{h} / \mathrm{kg}\end{array}$ \\
\hline $\begin{array}{l}\text { Flux basal } \ldots \ldots \ldots \ldots \ldots \ldots \ldots \\
\text { Flux modifié } \ldots \ldots \ldots \ldots \ldots \ldots \\
\text { CT }\end{array}$ & $\begin{array}{l}0,0168 \pm 0,0016(8) * \\
0,0101 \pm 0,0014(8)\end{array}$ & $\begin{array}{l}13,86 \pm 3,39(9) \\
20,40 \pm 3,70(9)\end{array}$ \\
\hline Signification ** & $p<0,01$ & $P<0,01$ \\
\hline
\end{tabular}

* Moyenne \pm erreur standard de la moyenne, nombre de déterminations entre parenthèses. ** Test employé $=$ test $t$ par paires de variables (Ostle, 1963). 
Une semaine après ultimobranchialectomie, le flux basal d'entrée de calcium au niveau branchial est inférieur à celui des témoins. L'opération provoque une diminution faible non significative du flux basal de sortie (tabl. 2).

TABLEAU 2

Effet de l'ulfimobranchialectomie sur les flux branchioux de calcium chez l'Anguille

\begin{tabular}{|c|c|c|}
\hline & $\begin{array}{c}\text { Flux d'entrée } \\
\mu \mathrm{Eq} / \mathrm{h} / \mathrm{kg}\end{array}$ & $\begin{array}{c}\text { Flux de sortie } \\
\mu \mathrm{Eq} / \mathrm{h} / \mathrm{kg}\end{array}$ \\
\hline $\begin{array}{l}\text { Flux basaux } \\
\text { Anguille témoin } \ldots \ldots \ldots \ldots \ldots \ldots \ldots \\
\text { Flux basaux } \\
\text { Anguille ultimobranchialectomisée } \ldots\end{array}$ & $\begin{array}{l}0,0168 \pm 0,0016(8) * \\
0,00725 \pm 0,0009(10)\end{array}$ & $\begin{array}{l}13,87 \pm 3,39(9) \\
11,54 \pm 1,62(12)\end{array}$ \\
\hline Signification $* * \ldots \ldots \ldots$ & $p<0,001$ & $p>0,05$ \\
\hline
\end{tabular}

* Moyenne \pm erreur standard sur la moyenne, nombre de déterminations entre parenthèses.

** Test employé = test par paires de variables (Ostle, 1963).

Enfin, chez ces animaux ultimobranchialectomisés, la calcitonine diminue le flux d'entrée ef augmente le flux de sortie (tabl. 3).

TABLEAU 3

Action de la CT sur les flux branchiaux de calcium chez l'Anguille après ultimobranchialectomie

\begin{tabular}{|c|c|c|}
\hline & $\begin{array}{c}\text { Flux d'entrée } \\
\mu \mathrm{Eq} / \mathrm{h} / \mathrm{kg}\end{array}$ & $\begin{array}{c}\text { Flux de sortie } \\
\mu \mathrm{Eq} / \mathrm{h} / \mathrm{kg}\end{array}$ \\
\hline $\begin{array}{l}\text { Flux basaux après ultimobranchialec- } \\
\text { tomie } \ldots \ldots \ldots \ldots \ldots \ldots \ldots \ldots \ldots . . . . . . . \\
\text { Flux basaux après ultimobranchialec- } \\
\text { tomie et addition in vitro de CT } \ldots \ldots\end{array}$ & $\begin{array}{l}0,00725 \pm 0,009(10)^{*} \\
0,00539 \pm 0,00058(10)\end{array}$ & $\begin{array}{l}11,54 \pm 1,62(12) \\
20,93 \pm 2,09(12)\end{array}$ \\
\hline Signification $^{* *} \ldots \ldots \ldots \ldots \ldots \ldots$ & $P<0,01$ & $P<0,01$ \\
\hline
\end{tabular}

* Moyenne \pm erreur standard sur la moyenne, nombre de déterminations entre parenthèses.

** Test t par paires de variables (Ostle, 1963).

Les effets de l'hormone sont donc qualitativement similaires à ceux obtenus chez les témoins. De plus, la suppression de l'hormone endogène semble modifier quantitativement la réponse de la branchie à la CT in vitro. En effet, le flux d'entrée qui était diminué de 39 p. 100 (par rapport au flux basal) chez les témoins ne l'est plus que de 29 p. 100 ; l'augmentation du flux de sortie par la CT qui était de 47 p. 100 est de 81 p. 100 après ultimobranchialectomie. Exprimées en valeur absolue la diminution du flux d'entrée (de $0,0066 \pm 0,001 \mu \mathrm{Eq} / \mathrm{h} / \mathrm{kg}$ à $0,0021 \pm 0,005 \mu \mathrm{Eq} / \mathrm{h} / \mathrm{kg}$ ) d'une part ef l'augmentation du flux de sortie (de $6,53 \pm 0,64 \mu \mathrm{Eq} / \mathrm{h} / \mathrm{kg}$ à $9,39 \pm$ $1,04 \mu \mathrm{Eq} / \mathrm{h} / \mathrm{kg})$ d'autre part sont significatives $(\mathrm{P}<0,001$ et $0,01>P>0,05)$. 


\section{Discussion.}

On sait que chez les Mammifères, la CT est hypocalcémiante. Son action inhibitrice de la résorption osseuse est maintenant bien établie (Milhaud et al., 1965 ; Friedman and Raisz, 1965 ; Gaillard, 1967 ; Foster et al., 1966) et un effet au niveau du rein est probable (Milhaud ef Job, 1966) ; Bartter ef al., 1969).

Chez les Poissons, le rôle de la calcitonine dans la régulation du métabolisme calcique est encore controversé probablement en raison du fait que l'action hypocalcémiante de cette hormone est difficile à démontrer. La calcémie est en effet le paramètre que la plupart des auteurs ont suivi : des résultats positifs ont été obtenus par Louw et al. (1967) sur le Poisson chat (Ictalurus melas) ainsi que par Chan ef al. (1968) sur l'Anguille (Anguilla anguilla) tandis que Pang et Pickford (1967) n'ont observé aucun effet de la CT sur la calcémie de Fundulus hétéroclitus. Nous-même, chez la Truite, n'avons pu mettre en évidence l'effet de la CT mammalienne au niveau sanguin, mais son effet sur l'os a été clairement démontré (Lopez et al., 1971). Chez l'Anguille maintenue en eau de mer, la CT synthétique de Saumon provoque une baisse significative de la calcémie, une atrophie du CUB et une importante stimulation de l'anabolisme osseux (Lopez ef al., 1976 ; Peignoux-Deville ef al., 1975).

L'ensemble des données expérimentales exposées ci-dessus montre nettement que la branchie d'Anguille est aussi un organe cible pour la calcitonine. En réduisant le flux d'entrée de calcium et surtout en augmentant le flux de sortie la CT tend, par ses actions au niveau branchial, à abaisser le taux de calcium sanguin. L'effet de l'ablation du CUB sur les flux de calcium branchiaux ne se manifeste qu'au niveau du flux d'entrée, ef d'une façon qui peut paraître contradictoire puisqu'elle le diminue. Nous supposons qu'il s'agif là d'un effet indirect, médié par les corpuscules de Stannius. Milet ef al. (1975) ont en effet montré qu'une semaine après l'ablation des corpuscules de Stannius, le flux d'entrée est augmenté par rapport à celui des témoins, alors que le flux de sortie est diminué. Une perfusion d'extrait de ces glandes diminue le flux d'entrée et augmente le flux de sortie (Milet ef al., en préparation). La diminution du flux d'entrée, constatée après ultimobranchialectomie serait due à une sécrétion accrue de l'hormone des corpuscules de Stannius, qui sont particulièrement activés eprès ablation du CUB (Lopez ef al., 1976).

La suppression de la CT endogène modifie l'intensité des réponses de la branchie à la CT : elle accroît l'action de cette hormone sur le flux de sortie et la diminue au niveau du flux d'entrée. Cette dissociation des effets de la CT sur les flux de calcium suggère une relative indépendance des mécanismes présidant aux transferts de l'ion calcium. Nous avons constaté au cours de nos expérimentations que la modification provoquée par la CT sur le flux d'entrée branchial de divers animaux est toujours proportionnelle au flux basal. Cette corrélation pourrait expliquer le fait que la CT diminue davantage le flux d'entrée chez les animaux témoins que chez les ultimobranchialectomisés, le flux d'entrée étant inférieur chez ces derniers.

Un travail récent effectué au laboratoire (Chartier et al., 1977) a montré que les cellules à chlorure étaient plus nombreuses et très activées après ablation des corpuscules de Stannius, opération qui provoque une entrée accrue de calcium à travers les branchies (Fontaine et al., 1972). Les cellules à chlorure, qui pourraient jouer un 
rôle similaire à celui des cellules tubulaires du rein et excréter des substances organiques et inorganiques (Motais et Garcia-Romeu, 1972) sont susceptibles d'être le siège du transport actif de calcium et sont sans doute impliquées dans les modifications de transport de calcium que nous venons de décrire. L'étude histologique des branchies est en cours.

Quoi qu'il en soit nos résultats montrent que, chez l'Anguille, le corps ultimobranchial, par l'intermédiaire de l'hormone qu'il sécrète, la calcitonine, contrôle le taux de calcium sanguin en agissant non seulement sur l'os, mais aussi sur l'épithélium branchial : il augmente le flux net qui est un flux négatif.

\section{Réunion Groupe Développement INRA/Productions animales Montpellier, 17-18 mai 1977.}

\section{Références}

BARTTER F. C., PAK C. Y. C., LIFSCHITZ M., EAST D., 1969. The role of calcium infusions and thyrocalcitonin in the treatment of metabolic bone diseases, cité par PAK C. Y. C., RURKIN B., CASPER A., 1970. Renal effects of thyrocalcitonin, 281-294. In TAYLOR S., FOSTER G. V. Calcifonin. Proc. 2nd inter. symp., Heineman, London.

BILLS C. E., HARRIS R. S., JONES J. H., KANOF A., KRAMER B., 1954. Vitamin D group, II, 131-266. In SEBRELL W. H. Jr, HARRIS R. S., The vitamins-chemistry, physiology, pathology. Acad. Press, N. Y., London.

CHAN D. K. O., 1972. Hormonal regulation of calcium balance in teleost fish. Gen. comp. Endocr., suppl. 3, 411-420.

CHAN D. K. O., CHESTER-JONES I., SMITH R. N., 1968. The effect of mammalian calcitonin on the plasma levels of calcium and inorganic phosphate in the european eel (Anguilla anguilla L.). Gen. comp. Endocr., 11, 243-245.

CHARTIER M. M., MILET C., LOPEZ E., LALLIER F., MARTELlY E., WARROT S., $1977 . \quad$ Modifications morphologiques, cytologiques et biochimiques de la branchie d'Anguilla anguilla $\mathrm{L}$. après ablation des corpuscules de Stannius. J. Physiol. Paris, 73, 23-26.

CHESTER-JONES I., HENDERSON I. W., BUTLER D. G., 1965. Osmoregulation in teleost fish with special reference to the european eel (Anguilla anguilla L.). V. Contrôles endocriniens dans le métabolisme de l'eau et des électrolytes. Arch. Anat. micros. Morph. exp., 54, 435-469.

FLESCHMAN W., 1947. Comparative physiology of the thyroid gland. Quart. Rev. Biol., 22, $119-140$.

FONTAINE M., 1964. Corpuscules de Stannius et régulation ionique $(\mathrm{Ca}, \mathrm{K}, \mathrm{Na})$ du milieu intérieur de l'Anguille (Anguilla anguille L.). C. R. Acad. Sci. Paris, 259, 875-878.

FONTAINE M., 1967. Intervention des corpuscules de Stannius dans l'équilibre phosphocalcique du milieu intérieur d'un poisson téléostéen, l'anguille. C. R. Acad. Sci. Paris, Série D, 264, 736-737.

FONTAINE M., DELERUE N., MARTELLY E., MARCHELIDON J., MILET C., 1972. Rôle des corpuscules de Stannius dans les échanges de calcium d'un poisson téléostéen, l'anguille (Anguilla anguilla L.) avec le milieu ambiant. C. R. Acad. Sci. Paris, Série D, 275, 1523-1528.

FOSTER G. V., DOYLE F. H., BORDIER P., MATRAJT H., 1966. Effect of thyrocalcitonin on bone. Lancet, 2, 1428.

FRIEDMAN J., RAISZ L. G., 1965. Thyrocalcitonin : Inhibitor of bone resorption in tissue culture Science, 150, 1465.

GAILLARD P. J., 1967. Bone culture studies with thyrocalcitonin. K. Ned. Akad. Wef Amsterdam, Proc. Ser. C., 70, 309.

HOAR W. S., 1951. Hormones in fish. In : Some aspects of the physiology of fish. Univ. Toronto, Biol. Ser., 59, 1-51.

LOPEZ E., CHARTIER-BARADUC M. M., DEVILLE J., 1971. Mise en évidence de l'action de la calcitonine porcine sur l'os de la truite Salmo gairdnerii soumise à un traitement déminéralisant. C. R. Acad. Sci., Paris, Série D, 272, 2600-2603. 
LOPEZ E., DEVILLE J., BAGOT E., 1968. Etude histophysiologique du corps ultimobranchial d'un téléostéen Anguilla anguilla L. au cours d'hypercalcémies expérimentales. C. R. Acad. Sci. Paris, Série D., 267, $1531-1534$.

LOPEZ E., PEIGNOUX-DEVILLE J., LALLIER F., MARTELLY E., MILET C., 1976. Effecis of calcitonin and ultimobranchialectomy (UBX) on calcium and bone metabolisms in the eel, Anguilla anguilla L. Calcif. Tiss. Res., 20, 173-186.

LOUW G. N., SUTTON W. S., KENNY A. D., 1967. Action of calcitonin in the teleost fish Ictalurus melas. Nature, 215, 888-889.

MILET C., MARTELLY E., FONTAINE M., 1975. Corpuscules de Stannius (CS) et flux de calcium au niveau des branchies perfusées d'Anguilla vulgaris. J. Physiol. Paris, 71, $141 \mathrm{~A}$.

MILHAUD G., JOB. J. C., 1966. Thyrocalcitonin : effect on idiopathic hypercalcemia. Science, 154, 794.

MILHAUD G., PERAULT A. M., MOUKHTAR M. S., 1965. Ełude du mécanisme de l'action hypocalcémiante de la thyrocalcitonine. C. R. Acad. Sci. Paris, 261, 813-816.

MOTAIS R., GARCIA-ROMEU F., 1972. Transport mechanisms in the teleostean gill and amphibian skin. Annu. Rev. Physiol., 34, 141-176.

ORIMO H., YAMAUCHI H., OHYAMA T., 1976. Biological and immunological properties of eel calcitonin, 157-158. Inter. Congr. Endocrinol., Hamburg, Fed. Rep. Germany, July 18-24,

OSTLE B., 1963. Statistics in research. lowa State Univ. Press.

PANG P. K. T., PICKFORD G. E., 1967. Failure of hog thyrocalcitonin to elicit hypocalcemia in the teleost fish Fundulus heteroclitus. Comp. biochem. Physiol., 21, 573-578.

PEIGNOUX-DEVILLE J., LOPEZ E., LALLIER F., MARTELLY-BAGOT E., MILET C., 1975. Responses of the ultimobranchial body in eels, Anguilla anguilla L. maintained in sea water and experimentally matured to injections of synthetic salmon calcitonin. Cell Tiss. Res., 164, 73-83.

PICKFORD G. E., 1953. A study of the hypophysectomized male killifish, Fundulus heferoclifus. Bull. Bingham. oceanogr. Coll., 14, 5-11.

STANNIUS H., 1839. Uber Nebennieren bei Knochenfischen. Arch. Anat. Physiol. Wiss. Med. 6, 97-101.

WATTS E. G., COPP D. H., DEFTOS L. J., 1975. Changes in plasma calcitonin and calcium during the migration of salmon. Endocrinology, 96, 214-218. 